\title{
Comment on "Effect of Extracorporeal Shockwave Therapy Versus Intra-articular Injections of Hyaluronic Acid for the Treatment of Knee Osteoarthritis"
}

\author{
Valter Santilli, PhD, Federica Alviti, PhD, Marco Paoloni, PhD, \\ Massimiliano Mangone, PhD, Andrea Bernetti, MD
}

Department of Physical Medicine and Rehabilitation, Sapienza University of Rome, Rome, Italy

In response to the original article: Ann Rehabil Med 2017;41(5):828-835

\section{Dear Editor,}

We read with great interest the study by Lee et al. titled "Effect of Extracorporeal Shockwave Therapy Versus Intra-articular Injections of Hyaluronic Acid for the Treatment of Knee Osteoarthritis" [1]. The authors were able to evaluate and compare the effects and outcomes of the extracorporeal shock wave therapy and intraarticular injections of hyaluronic acid in patients with knee osteoarthritis. This research is considered relevant and interesting, especially because it is noted that the authors compare an invasive therapy, such the intraarticular injections, with the administration of an extracorporeal show wave therapy, which is notably a kind of therapy that does not involve the interruption of the integrity of the skin. It is noted that the results from the study are promising from a clinical aspect; however, we would like to underline how the studying and review of the patient's clinical status with a visual analogue scale, Western Ontario and McMaster Universities Osteoarthritis Index (WOMAC), Lequesne index, 40-m fast-paced walk test, and stair-climb test should be supported by biomechanical information obtained from the precise study of walking pattern using a gait analysis. From this point of view, it is our determination that a gait analysis evaluation should be considered as an objective and useful instrumentation in order to have more data that could identify differences in the walking cycle's sub-phases, before and after any kind of treatment for lower limb's osteoarthritis. In our opinion, the work performed by Lee et al. [1] is relevant, important and useful, however, with would like to ask to the authors if they have the intention to provide results after a longer follow up time frame to be comprehensively reviewed in a further study. We recognize and agree that the subject of knee osteoarthritis is a theme currently representing an emerging topic for its increasing incidence, and it is well known that knee osteoarthritis is a chronic disease that could lead to important long-lasting disability. Therefore, knowing the

Corresponding author: Andrea Bernetti

Department of Physical Medicine and Rehabilitation, Sapienza University of Rome, Piazzale Aldo Moro, 5, Rome, Italy. Tel: +39-320-946-7954, Fax: +39-64-991-4846, E-mail: andrea.bernetti@uniroma1.it

ORCID: Valter Santilli (http://orcid.org/0000-0002-2547-6801); Federica Alviti (http://orcid.org/0000-0002-0811-8009); Marco Paoloni (http://orcid. org/0000-0002-0947-9711); Massimiliano Mangone (http://orcid.org/0000-0002-5235-0948); Andrea Bernetti (http://orcid.org/0000-0002-0204-9831).

(c) This is an open-access article distributed under the terms of the Creative Commons Attribution Non-Commercial License (http://creativecommons.org/ licenses/by-nc/4.0) which permits unrestricted noncommercial use, distribution, and reproduction in any medium, provided the original work is properly cited. Copyright (C) 2018 by Korean Academy of Rehabilitation Medicine 
long-term effects and outcomes of a therapy for osteoarthritis could be more interesting, and we would read more on this topic if provided the opportunity with new studies on the issue. Additionally, the use of a gait analysis should be desirable during the diagnosis and followup from different pathologies that could affect the walking cycle, not only in orthopedics diseases, but also in a neurologic condition such as hemiplegia [2] or bilateral spastic cerebral palsy [3]. Actually, the relevant therapies dedicated to osteoarthritis should be not directed only on pain management and relief as the main target, but any therapeutic strategy should also involve techniques that are specifically focused on the patient's functional status and disability. From these considerations, we appreciate the author's choice to include the measurement of the 40-m fast-paced walk test, and stair-climb test in their study, but once again, we would like to underline the importance of the use an objective tool as a relevant gait analysis, in order to guarantee a precise evaluation that could be used also during this kind of testing or to accurately measure other motor tasks [4].

\section{REFERENCES}

1. Lee JK, Lee BY, Shin WY, An MJ, Jung KI, Yoon SR. Effect of extracorporeal shockwave therapy versus intraarticular injections of hyaluronic acid for the treatment of knee osteoarthritis. Ann Rehabil Med 2017;41: 828-35.

2. Kim HD, Kim JG, Jeon DM, Shin MH, Han N, Eom $\mathrm{MJ}$, et al. Analysis of vertical ground reaction force variables using foot scans in hemiplegic patients. Ann Rehabil Med 2015;39:409-15.

3. Kim DJ, Park ES, Sim EG, Kim KJ, Kim YU, Rha DW. Reliability of visual classification of sagittal gait patterns in patients with bilateral spastic cerebral palsy. Ann Rehabil Med 2011;35:354-60.

4. Eden MM, Tompkins J, Verheijde JL. Reliability and a correlational analysis of the 6MWT, ten-meter walk test, thirty second sit to stand, and the linear analog scale of function in patients with head and neck cancer. Physiother Theory Pract 2018;34:202-11. 